\title{
Research on a Laser Ring Induced by a Metal Wire
}

\author{
Yu Feng, Guang Wang, Min Zhao*
}

School of Physics Science and Engineering, Tong Ji University, Shanghai, China

Email address:

minzhaotj@tongji.edu.cn (Min Zhao)

${ }^{*}$ Corresponding author

To cite this article:

Yu Feng, Guang Wang, Min Zhao. Research on a Laser Ring Induced by a Metal Wire. American Journal of Physics and Applications. Vol. 5, No. 2, 2017, pp. 29-34. doi: 10.11648/j.ajpa.20170502.14

Received: April 6, 2017; Accepted: June 5, 2017; Published: June 15, 2017

\begin{abstract}
When the metal wire is irradiated with a beam of laser, a light ring will appear on the screen. The formation mechanism of the light ring is discussed in this article. It is also discussed how various parameters, including the shape of the laser beam, the laser diameter, the wire diameter, the wire roughness, and the incidence angle, influence the ring through computational simulation and experiments simultaneously. Our calculation is in good agreement with experimental results.
\end{abstract}

Keywords: Light Ring, Reflection, Conic Curve

\section{Introduction}

Being given a section of metal wire and a beam of laser, a light ring can be created on the screen. Questions about how this phenomenon generates and by what means the parameters of the wire and the laser beam affect the ring's shape, attracted many people's attention when first proposed on the 21 st IYPT [1]. There are several papers discussing experimental results and qualitative interpretations of the laser ring phenomenon. But none of them could give a quantitative interpretation [2-4]. Some other papers discuss a lot about diffraction [5-8], which totally deviates the principle of the laser ring phenomenon.

This paper introduces our physics model and quantitative analysis in detail. With computational simulation, we reproduced a set of light rings and got a perfect relationship between the laser ring phenomenon and various parameters, which are in good agreement with our experiments.

\section{Theoretical Analysis}

\subsection{Analysis of a Thin Plane Laser's Incidence}

Generally, the reflecting surface of the laser and metal wire is the intersection surface of two cylinders. To simplify the model, we firstly focused on the reflection surface which is created by a thin plane laser irradiated on the metal wire. As in FIG1 (a), the intersection of the laser plane and metal wire is an ellipse. The reflection light on each point in the intersection ellipse changes its direction when the reflection plane changes. So the incident angle of the laser isn't a constant. FIG1 (b) shows that the incident angle is minimum at the middle point of the elliptical intersection, and the incident direction of the laser beam doesn't change at the edge of the intersection. As for other points of the intersection, the incident angle lies between the minimum angle and $90^{\circ}$.

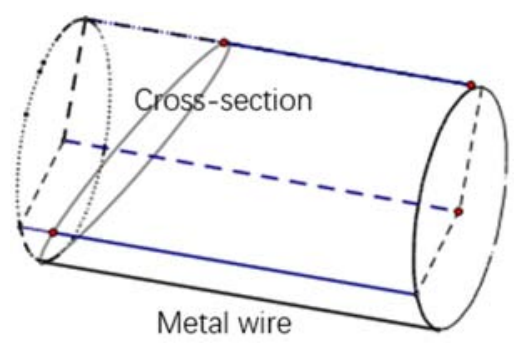

(a)

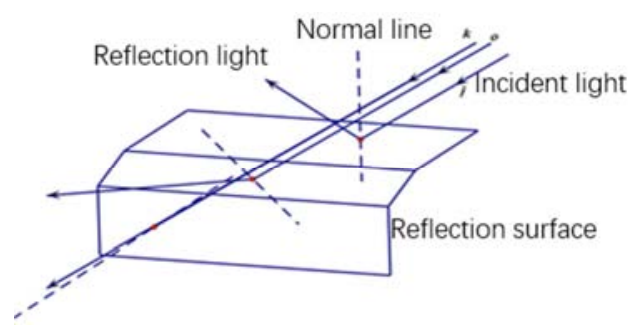

(b)

Figure 1. Reflecting lights path of a laser. 
All the reflected lights are casted on the screen, as shown in FIG2 (a). The reflection angle of the laser in the middle point of the intersection is the minimum angle marked as $\theta_{\text {min }}$, and the distance from the middle point to the screen is $\mathrm{s}$.

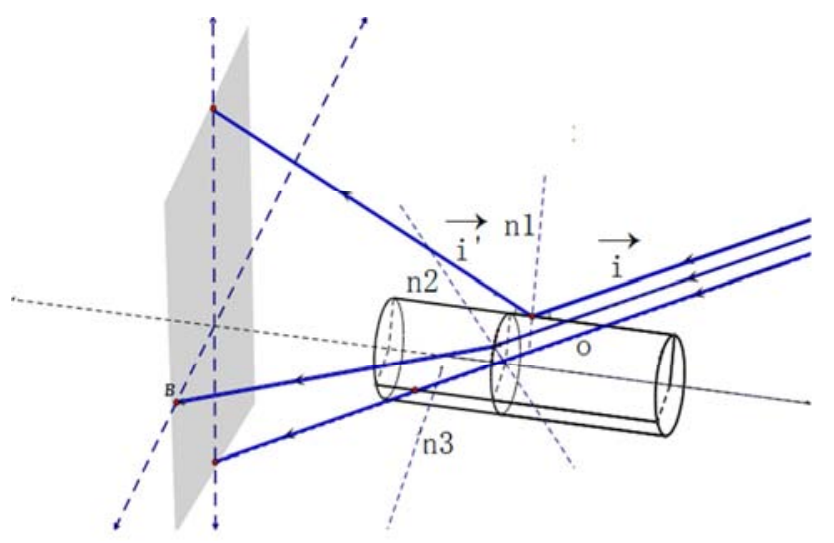

(a)

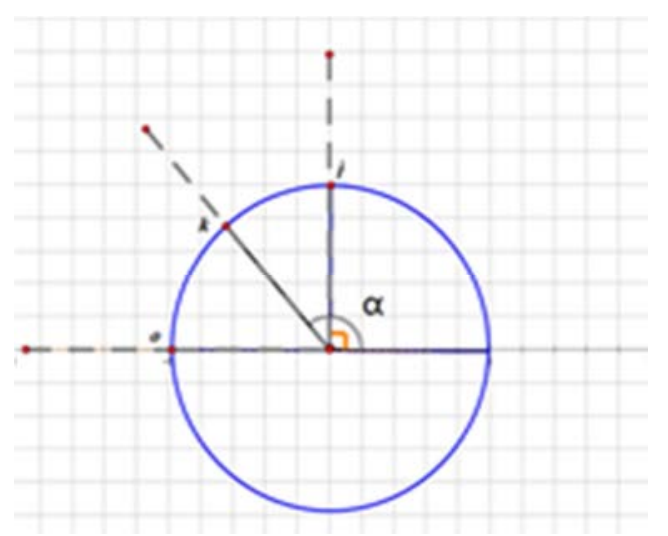

(b)

Figure 2. Reflected lights create a light ring.

As is shown in FIG 2(b), the projected curve of the elliptical intersection on the screen is a set of reflection surfaces' normal lines, called as a normal plane. Rotary coordinates are established on our normal plane. $\alpha$ is the angle between a normal line and the horizontal axis. The radius of metal wire is $r$, so the direction vector $\underset{i}{\rightarrow}$ and normal vector $\underset{n}{\rightarrow}$ of incident laser are obtained.

$$
\begin{aligned}
& \tilde{i}=\left(0, \mathrm{r},-\frac{r}{\tan \left(\theta_{\text {min }}\right)}\right) \\
& \tilde{n}=(\operatorname{rcos} \alpha, \operatorname{rsin} \alpha, 0)
\end{aligned}
$$

Using the reflection matrix $[9,10]$, we can get the reflection

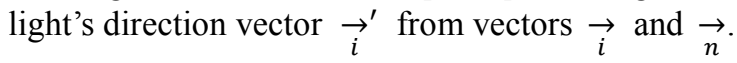

$$
\tilde{i^{\prime}}=\left[\begin{array}{ccc}
1-2 n_{1}^{2} & -2 n_{2} n_{1} & -2 n_{3} n_{1} \\
-2 n_{1} n_{2} & 1-2 n_{2}^{2} & -2 n_{3} n_{2} \\
-2 n_{1} n_{3} & -2 n_{2} n_{3} & 1-2 n_{3}^{2}
\end{array}\right]_{i^{T}}^{T}
$$

Then the intersection point of the reflection light is obtained in (3). And the reflection image equations can be easily obtained as following.

$$
\left\{\begin{array}{l}
x=\left(r+s \cdot \tan \left(\theta_{\min }\right)\right) \cdot \cos \beta \\
\mathrm{y}=\left(r+\mathrm{s} \cdot \cot \left(\theta_{\min }\right)\right) \cdot \sin \beta \\
\mathrm{z}=\mathrm{s}
\end{array}\right.
$$

$\beta$ is the central angle of the halo on imaging screen. MATLAB 2013a is used to give the simulation result shown in FIG 3.

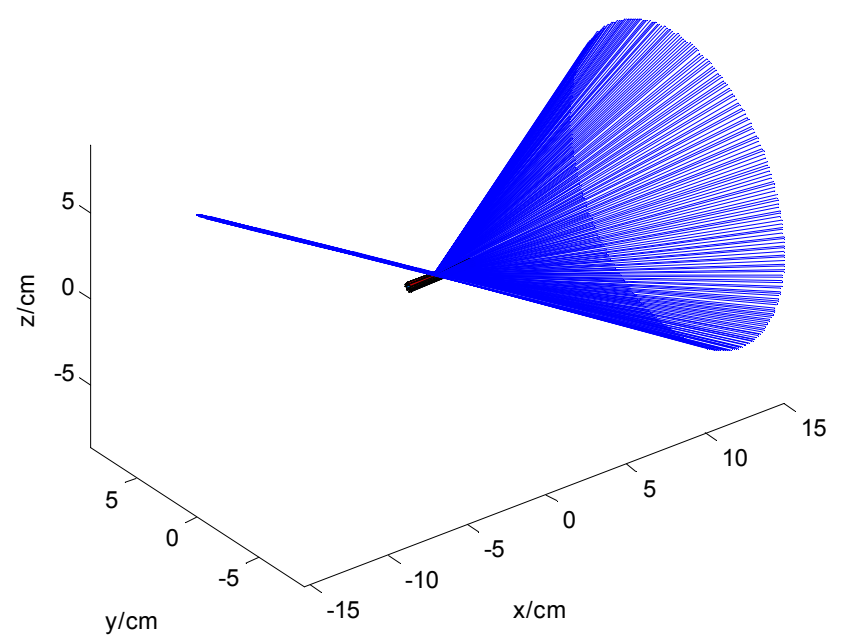

Figure 3. Simulation light ring created by reflection lights.

When a planar laser is irradiated on a horizontal metal wire, the reflectance image on the screen which is perpendicular to wire will be a circle. And the ring's radius depends on the distance from the incident point to the imaging screen.

All the discussion above is founded on the basis of a thin plane laser beam. But normally the shape of a laser beam is not a plane, so we try to find out what will happen when it comes to be a cylinder-shaped laser.

\subsection{Analysis of a Cylinder Laser's Incidence}

In the general case, the reflection plane is the intersection plane of two different cylinders. We divided the cylinder-shaped laser into various thin laser planes. The minimum width of these planes is supposed to be zero, and the diameter section of the laser cylinder has the maximum width.

The coordinate is established based on the middle point of the intersection of the thin laser plane and the metal wire, respectively, to obtain various reflection vectors. After calculating intersection points of reflection light rays, the reflectance image of a cylinder laser irradiated on a metal wire is obtained.

It is worthy noted that when the laser beam cannot cover the upper surface of metal wire, the reflectance image is no longer a total circle as before but a part of it. Thus, the cylinder laser's reflectance image is a combination of many different circles and incomplete circles, as is shown in FIG4. It shows as an 
uneven conical shell in three-dimensional space. The shell's maximum width and minimum width is calculated as following:

$$
\left\{\begin{array}{l}
w_{\text {max }}=2 R \tan \left(\theta_{\text {min }}\right) \\
\mathrm{w}_{\text {min }}=2 \sqrt{R^{2}-r^{2}}
\end{array}\right.
$$

$R$ is the radius of laser.

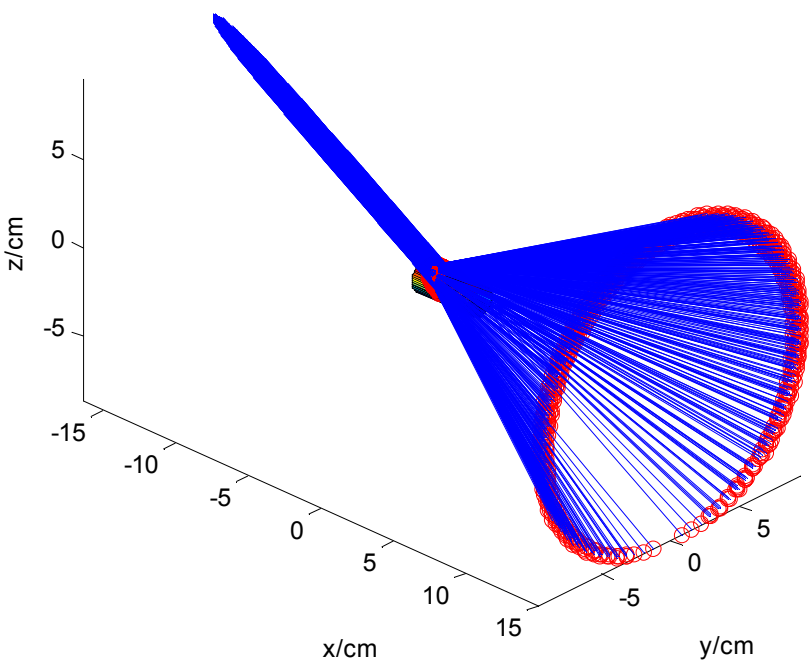

(a)

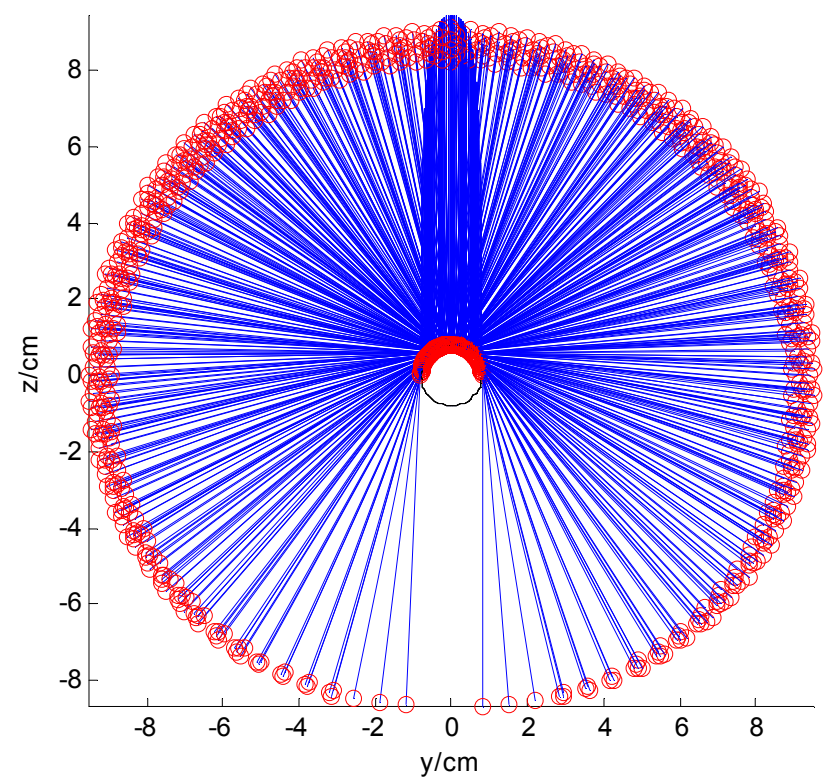

(b)

Figure 4. Simulation reflectance image of a cylinder laser.

\section{Experimental Results and Discussion}

\subsection{Relationship Between Light Ring's Radius and Related Parameters}

Different kinds of metal wires were changed and different radius of laser beams were used in our experiments. The parameters of various light rings can be measured by the grid paper. The experiment device is shown in FIG 5.

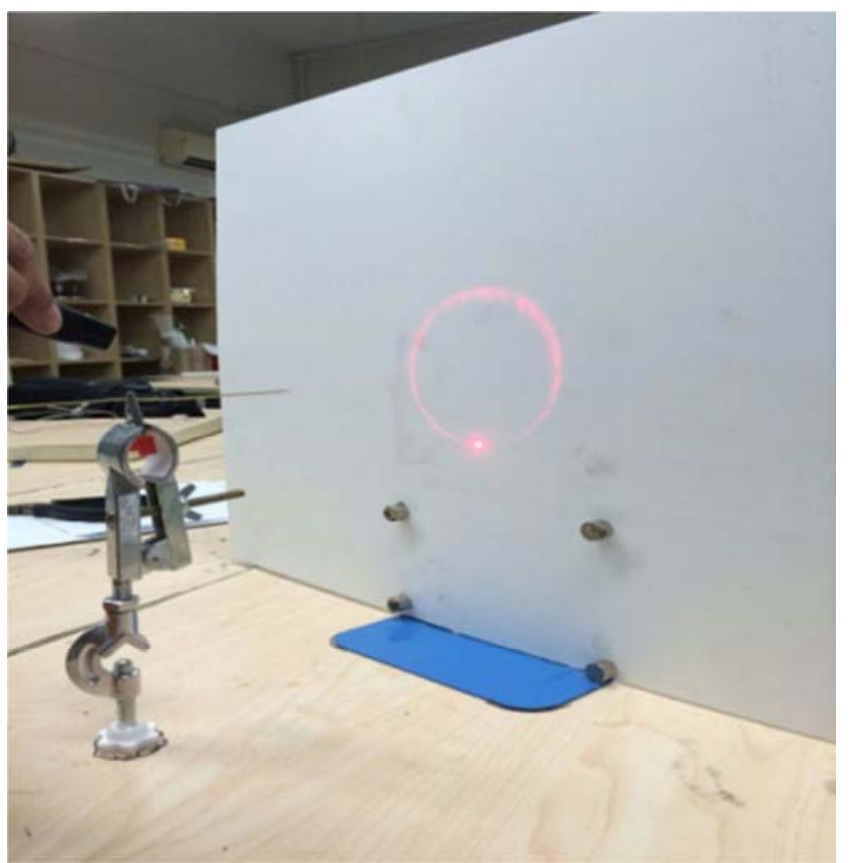

(a)

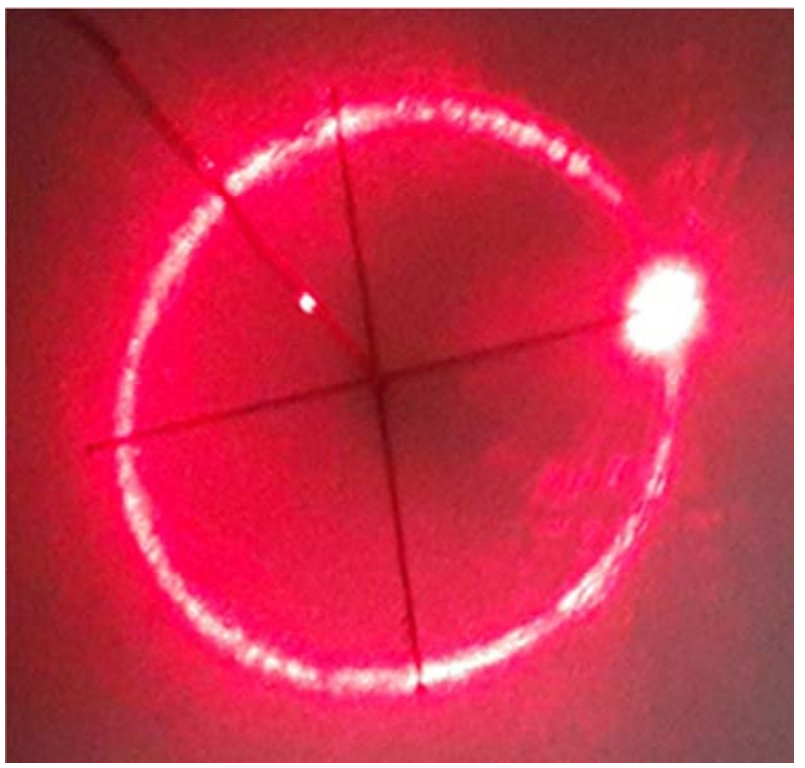

(b)

Figure 5. The experimental device and the phenomenon.

The radius of the light ring depends on the wire's radius and the laser's incident angle, as is shown in Eq (4). So we changed the incident angle and the metal wire's radius, measured the distance between the middle point of the light ring band and the circle center, plotted relationship curves of various parameters to compare with theoretical curves. FIG6 (a) shows the relationship between the ring's radius and the laser's incident angle. FIG6 (b) shows the relationship between the ring's radius and the metal wire's radius. 


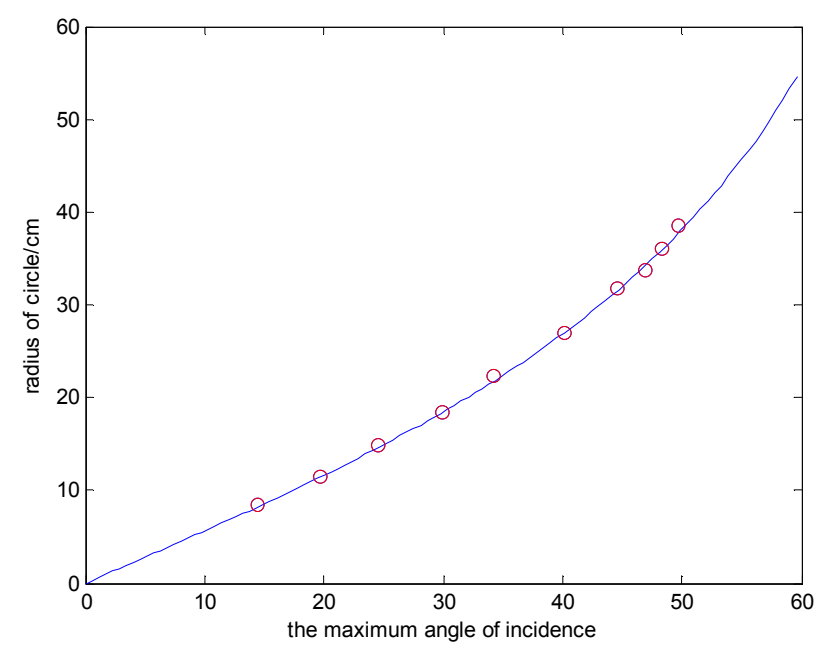

(a)

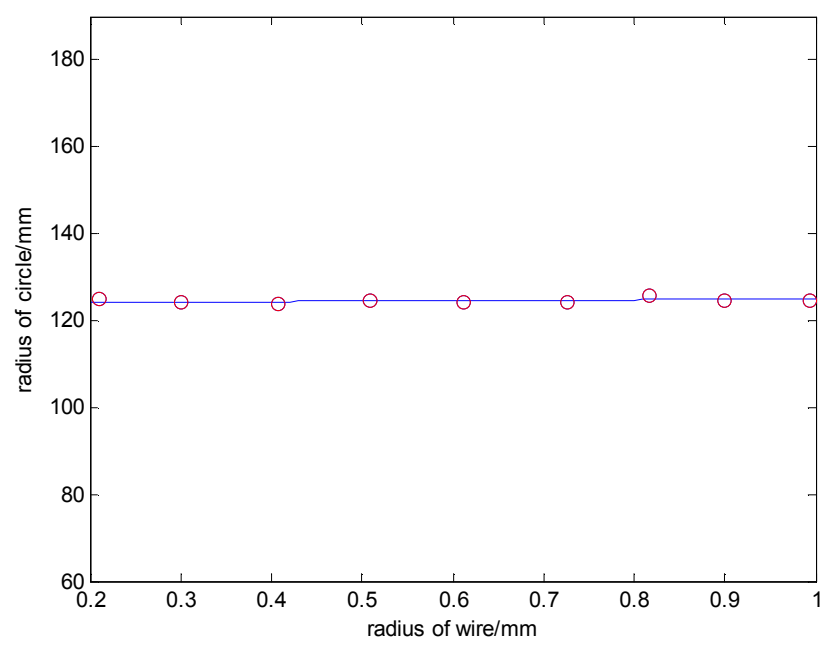

(b)

Figure 6. Light ring's radius changes with the laser's incident angle and the metal wire's radius.

The wire radius has little effect on the light ring, as is shown in FIG 6(b). That's because the light ring's radius depends both on the distance from the incident point to the screen and the metal wire radius. Generally, the wire radius is in milimeter's level, but the distance is in decimeter's level, so the effect caused by different wire's radiuses is negligible.

\subsection{Analysis of Incomplete Circle's Formation}

Sometimes there are gaps in the light ring. We also made simulation to give an explanation.

With simulation result in FIG 7(a), we can find a dark area with no reflectance laser on the back of the metal wire. In experiments, the laser beams without being reflected will produce a dark area and light spots as shown in FIG 7(b).

When the laser radius is less than the metal wire radius, different gaps will appear as is shown in FIG 7(c). It's because the laser cannot cover all the wire's upper surface. Supposing that the wire's radius is $0.8 \mathrm{~mm}$, we changed the incident laser's radius and got the transformation process from an incomplete circle to a complete one.

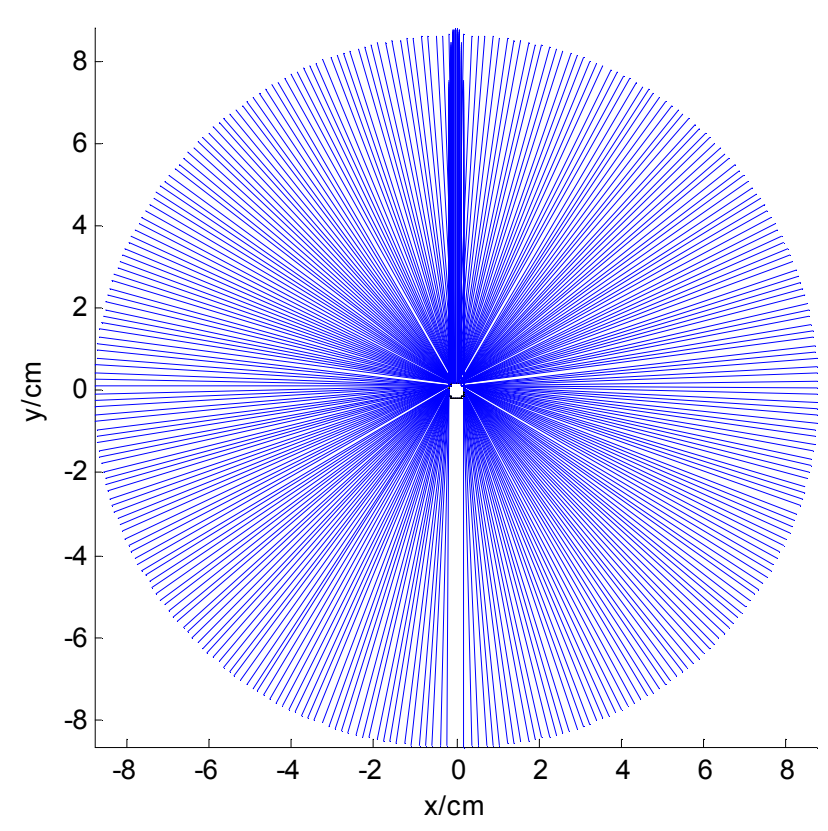

(a)

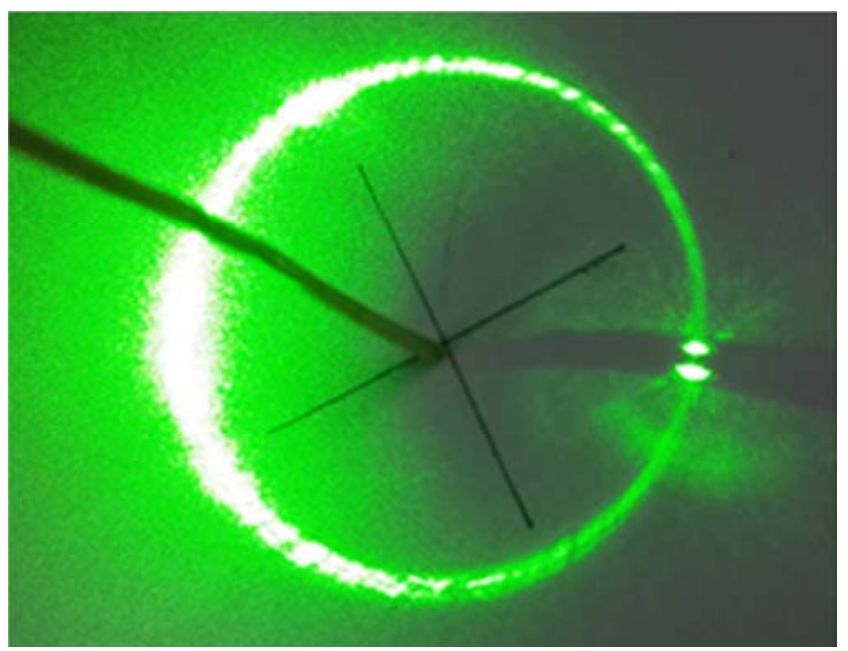

(b)

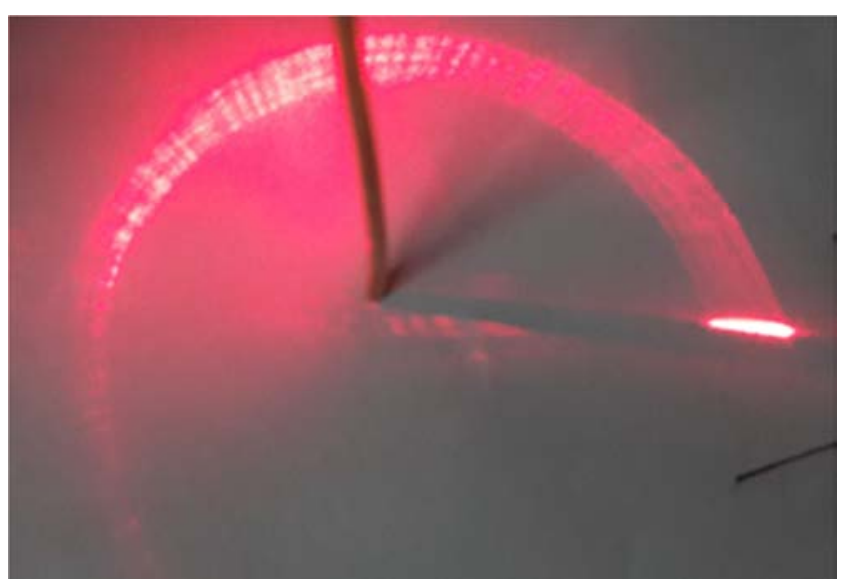

(c)

Figure 7. Simulation and experimental results of the ring gap. 


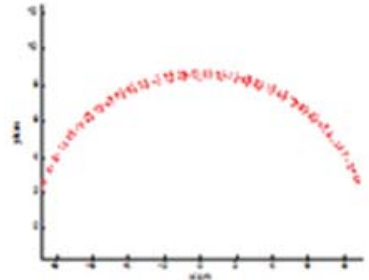

$\mathrm{R}=0.5 \mathrm{~mm}$

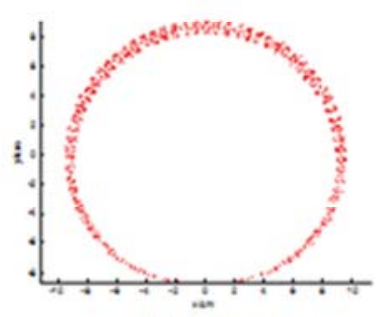

$\mathrm{R}=0.8 \mathrm{~mm}$

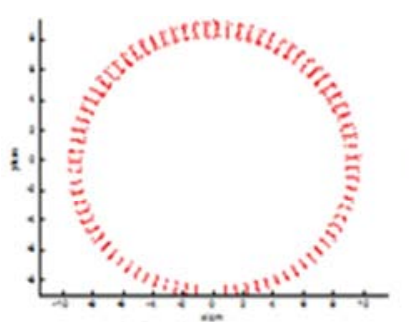

$\mathrm{R}=1.0 \mathrm{~mm}$
$\mathrm{R}=0.6 \mathrm{~mm}$

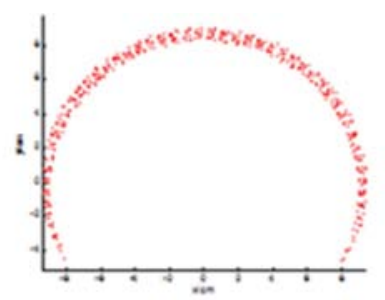

$\mathrm{R}=0.7 \mathrm{~mm}$

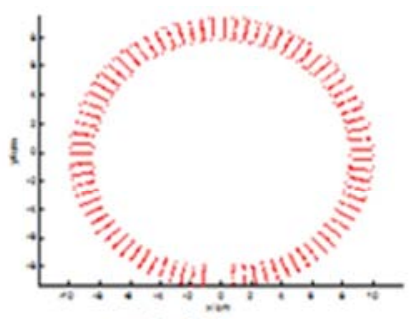

$\mathrm{R}=2.5 \mathrm{~mm}$

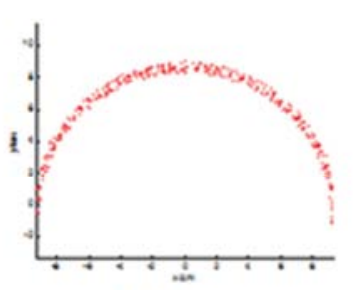

Figure 8. Simulation results of different incident laser radius.

It is shown in FIG 8 that when the laser's radius is smaller than the wire, the imaging ring is incomplete. On the contrary, when the laser's radius becomes bigger, the imaging ring will be a whole ring band with non-uniform thickness, and the non-uniformity will decrease as the radius of laser increasing.

\subsection{Effects of Wire's Roughness on the Ring}

In our experiment, the reflection isn't always a specular reflection because the roughness of metal wires has influence on the imaging formation, so we did some research on the roughness by Monte Carlo simulation. The reflecting surface is treated as a set of small mirror surfaces, and regarded the randomness of these mirror surfaces as roughness, so that the slight random number can be added to the normal vector of these mirror surfaces to simulate roughness. In this way, a normal vector of one point in the reflection lights intersection is got as following:

$$
\tilde{n}=((\mathrm{r}+\operatorname{rand} 1) \cos (\alpha+\operatorname{rand} 2),(\mathrm{r}+\text { rand } 1) \sin (\alpha+\text { rand } 2), 0+\text { rand } 3)
$$

Rand1, rand 2 and rand 3 represent the slight random number and form three directions respectively. So, a simulation reflecting image considering roughness is obtained in FIG 9 by $\mathrm{Eq}(1)$ and $\mathrm{Eq}(3)$.

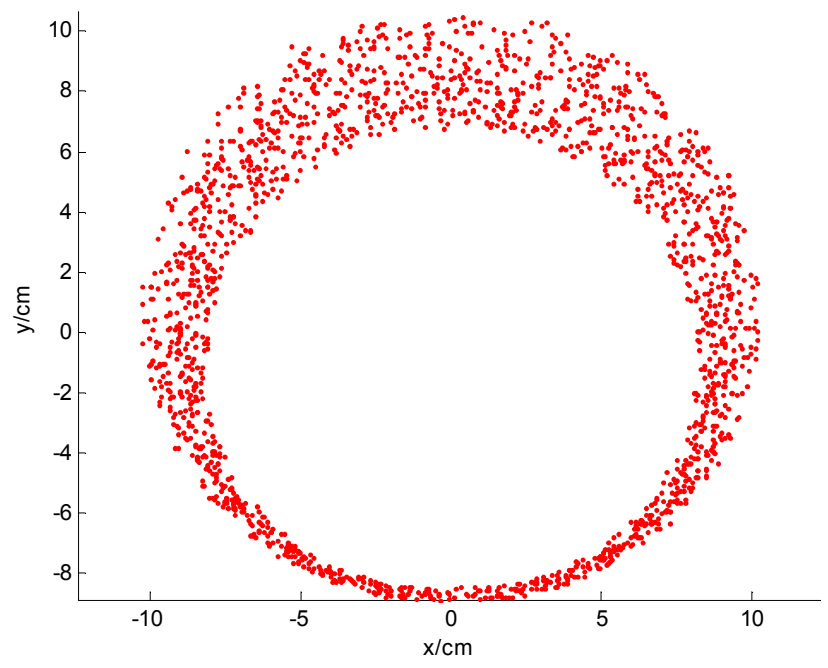

(a)

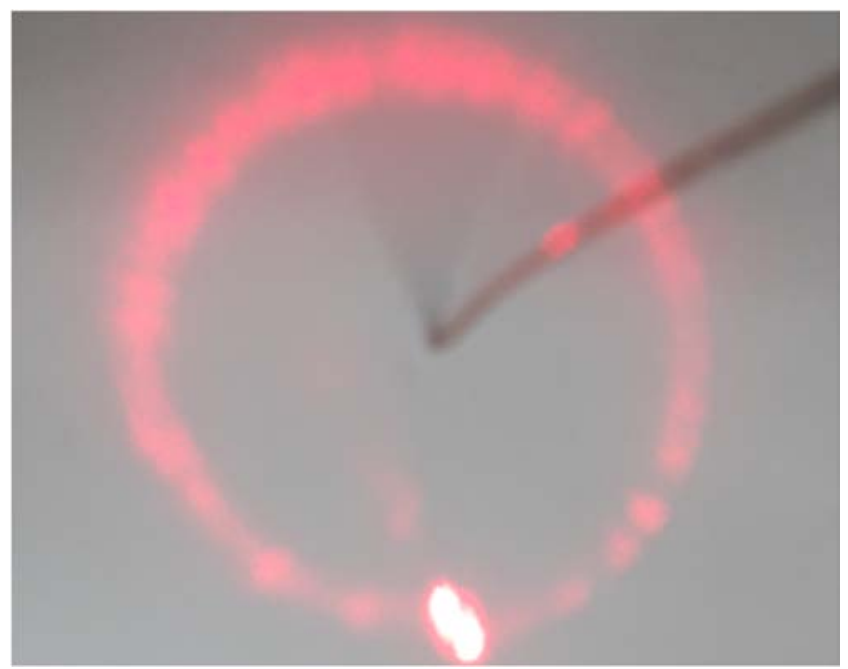

(b)

Figure 9. Simulation and experimental results with roughness being considered.

In FIG 9 we can easily find the imaging ring changes with the roughness of wire, and the width of the light ring increases if roughness increases.

\section{Conclusion}

In this paper, a reflecting model is established to explain why a light ring appears when irritating the metal wire with a laser beam. It is also discussed how various parameters, including the shape of the laser beam, the laser diameter, the wire diameter, the wire roughness, and the incidence angle, influence the ring through visual computational simulation and experiments simultaneously. When the laser irradiates on a rough metal wire, the ring will act as an uneven conical shell. Our calculation is in good agreement with the experimental results. 


\section{References}

[1] http://www.sogou.com/link?url=DSOYnZeCC_rL-beRucsA2k nsNhqzagA_\&query=iypt

[2] Junyi Zhu, Yabin Zhu, "Light circle and relevant parameters," Physics Experimentation, vol. 35(9), pp. 37-39

[3] Jianlin Zhao, Dexing Yang, "Spatial Light-Cone Induced by a Cylinder,” Acta Physica Sinica, vol. 51(9), pp. 1974-1977

[4] Guoye Guan, Jiajian Huang, Fang Lin, "Circle of Light Generated by Cylindrical Wire Obliquely Illuminated by Laser," Applied Physics, Vol 7(2), pp.43-53

[5] Keller, J. B., "Geometrical Theory of Diffraction," Journal of the Optical Society of America, Vol52, pp.390-405

[6] Keller, J. B. and Ahluwalia, D. S., "Diffraction by a Curved Wire," Siam Journal on Applied Mathematics, Vol20, pp.390-405

[7] Greenler, R. G., Hable, J. W. and Slane, P. O., "Diffraction around a Fine Wire: How Good is the Single-Slit Approximation?" American Journal of Physics, Vol58, pp. 330-331

[8] Tang, W., Zhou, Y. and Zhang, J., "Improvement on Theoretical Model for Thin-Wire and Slot Measurement by Optical Diffraction," Measurement Science \& Technology, Vol10, pp.119-123

[9] Yongkang Guo, Optics, Beijing: Higher Education Press, 2012

[10] Haidong Guo, "Three Means of Expression of Reflection Theorem", Jounral of Yunnan Normal University, vol. 20(1), pp. $57-60$ 